\title{
Phase diagram of polymer blends in confined geometry
}

\author{
M. Müllera*, K. Binder and E.V. Albano ${ }^{\mathrm{b}}$ \\ anstitut fiir Physik, WA 331, Johannes Gutenberg Universität, D-55099 Mainz, \\ Germany
}

bINIFTA, Universidad de La Plata, C.C. 16 Suc. 4. 1900 La Plata, Argentina

Within self-consistent field theory we study the phase behavior of a symmetrical binary $A B$ polymer blend confined into a thin film. The film surfaces interact with the monomers via short range potentials. One surface attracts the $A$ component and the corresponding semi-infinite system exhibits a first order wetting transition. The surface interaction of the opposite surface is varied as to study the crossover from capillary condensation for symmetric surface fields to the interface localization/delocalization transition for antisymmetric surface fields. In the former case the phase diagram has a single critical point close to the bulk critical point. In the latter case the phase diagram exhibits two critical points which correspond to the prewetting critical points of the semi-infinite system. Only below a triple point there is a single two phase coexistence region. The crossover between these qualitatively different limiting behaviors occurs gradually, however, the critical temperature and the critical composition exhibit a non-monotonic dependence on the surface field.

The dependence of the phase behavior for antisymmetric boundaries is studied as a function of the film thickness and the strength of the surface interactions. Upon reducing the film thickness or decreasing the strength of the surface interactions we can change the order of the interface localization/delocalization transition from first to second.

The role of fluctuations is explored via Monte Carlo simulations of a coarse grained lattice model. Close to the (prewetting) critical points we observe 2D Ising critical behavior. At lower temperatures capillary waves of the $A B$ interface lead to a pronounced dependence of the effective interface potential on the lateral system size.

(O) 2001 Elsevier Science B.V. All rights reserved.

\section{INTRODUCTION}

Confining a binary mixture gives rise to a rich interplay between wetting and miscibility behavior.[1-4] In a porous material or a slit-like pore with identical surfaces the critical point of the mixture is shifted away from its bulk value to lower temperatures and higher compositions of the component preferred by the walls.[4] The phenomenon of capillary condensation which occurs if the two confining boundaries are symmetric is well-known.[5] In some applications (e.g., coatings or dielectrics), however, the surfaces of the film (e.g., a solid substrate and vacuum) interact very differently with the constituents of the mixture.

"E-mail: Marcus.MuellerQuni-mainz.de 
In the opposite limit of antisymmetric walls, i.e., one wall attracts the $A$-component with exactly the opposite strength than the other wall the $B$-component, an interface localization/delocalization transition[6-10] occurs close to the wetting transition of the semi-infinite system.

We study the dependence of the phase behavior of a polymer mixture confined into a thin film on the surface interactions and film thickness via self-consistent field calculations[11-13] and Monte Carlo simulations.[14] Our paper is arranged as follows: In the next section we describe our mean field calculations and discuss the dependence of the phase diagram on the monomer-wall interactions. Then we investigate the effect of fluctuations via simulations of a coarse grained lattice model. The paper closes with a discussion of the anticipated chain length dependence and an outlook on experimental systems.

\section{SELF-CONSISTENT FIELD CALCULATIONS}

We calculate the phase behavior of a confined $A B$ mixture within the self-consistent field theory of Gaussian polymers.[15,16] The film comprises a volume $V_{0}=\Delta_{0} \times L \times L$. $\Delta_{0}$ denotes the film thickness, while $L$ is the lateral extension of the film. The density at the film surfaces decreases to zero in a boundary region of width $\Delta_{w}$ according to[17]

$\Phi_{0}(x)= \begin{cases}\frac{1-\cos \left(\frac{\pi x}{\lambda_{w}}\right)}{2} & ; 0 \leq x \leq \Delta_{w} \\ 1 & ; \Delta_{w} \leq x \leq \Delta_{0}-\Delta_{w} \\ \frac{1-\cos \left(\frac{\pi\left(\Delta_{0}-x\right)}{\Delta_{w}}\right)}{2} & ; \Delta_{0}-\Delta_{w} \leq x \leq \Delta_{0}\end{cases}$

where $\Phi_{0}$ denotes the ratio of the monomer density and the value $\rho$ in the middle of the film. The thickness $\Delta$ of an equivalent film with constant monomer density $\rho$ is $\Delta=\Delta_{0}-\Delta_{w}$. We choose $\Delta_{w}=0.15 R_{e},[17]$ where $R_{e}$ is the end-to-end distance. Both surfaces interact with the monomer species via a short range potential $H$ :

$H(x)= \begin{cases}\frac{4 \Lambda_{1} R_{e}\left\{1+\cos \left(\frac{\pi x}{\delta_{w}}\right)\right\}}{\Delta_{w}} & ; 0 \leq x \leq \Delta_{w} \\ 0 & ; \Delta_{w} \leq x \leq \Delta_{0}-\Delta_{w} \\ \frac{4 \Lambda_{2} R_{w}\left\{1+\cos \left(\frac{\pi\left(\Delta_{0}-x\right)}{\Delta_{w}}\right)\right\}}{\Delta_{w}} & ; \Delta_{0}-\Delta_{w} \leq x \leq \Delta_{0}\end{cases}$

$H>0$ is attractive for the $A$ monomers and repulsive for the $B$ species. The normalization of the surface fields $\Lambda_{1}$ and $\Lambda_{2}$, which act on the monomers close to the left and the right surface, is chosen such that the integrated interaction energy between the surface and the monomers is independent of the width of the boundary region $\Delta_{w}$.

$A$ and $B$ polymers contain $N$ monomers and are structurally symmetric. The polymer conformations $\left\{\mathbf{r}_{\alpha}(\tau)\right\}$ determine the microscopic $A$ monomer density

$\hat{\Phi}_{A}(\mathbf{r})=\frac{N}{\rho} \sum_{\alpha=0}^{n_{A}} \int_{0}^{1} \mathrm{~d} \tau \delta\left(\mathbf{r}-\mathbf{r}_{\alpha}(\tau)\right)$

where the sum runs over all $n_{A} A$ polymers in the system and $0 \leq \tau \leq 1$ parameterizes the contour of the Gaussian polymer. A similar expression holds for $\overline{\hat{\Phi}}_{B}(\mathbf{r})$. With this 
definition the semi-grandcanonical partition function takes the form:

$$
\begin{aligned}
\mathcal{Z} \sim & \sum_{n_{A}=1}^{n} \frac{e^{+\Delta \mu n_{A} / 2 k_{B} T}}{n_{A} !} \frac{e^{-\Delta \mu n_{B} / 2 k_{B} T}}{n_{B} !} \int \mathcal{P}_{A}[\mathbf{r}] \int \mathcal{P}_{B}[\mathbf{r}] \delta\left(\Phi_{0}-\hat{\Phi}_{A}-\hat{\Phi}_{B}\right) \\
& \times \exp \left(-\rho \int \mathrm{d}^{3} \mathbf{r}\left\{\chi \hat{\Phi}_{A} \hat{\Phi}_{B}-H\left(\hat{\Phi}_{A}-\hat{\Phi}_{B}\right)\right\}\right)
\end{aligned}
$$

where $n=n_{A}+n_{B}$ and $\Delta \mu$ represents the exchange potential between $A$ and $B$ polymers. The functional integral $\mathcal{P}$ sums over all conformations of the polymers with the statistical weight $\exp \left(-\frac{3}{2 R_{e}^{2}} \int_{0}^{1} \mathrm{~d} \tau\left(\frac{\mathrm{d} r}{\mathrm{~d} r}\right)^{2}\right)$ of a non-interacting Gaussian polymer. The second factor enforces the monomer density profile to comply with Eq.(1) (incompressibility). The Boltzmann factor in the partition function incorporates the thermal repulsion between unlike monomers, which is described by the Flory-Huggins parameter $\chi$, and the interactions between monomers and surfaces.

In mean field approximation the free energy is obtained as the extremum of the semigrandcanonical free energy functional:

$$
\begin{aligned}
\frac{\mathcal{G}}{n k_{B} T} \equiv+\ln & \frac{n}{V_{0}}-\ln \mathcal{Q}+\frac{1}{V} \int \mathrm{d}^{3} \mathbf{r} \chi N \Phi_{A} \Phi_{B}-H N\left\{\Phi_{A}-\Phi_{B}\right\} \\
& -\frac{1}{V} \int \mathrm{d}^{3} \mathbf{r}\left\{W_{A} \Phi_{A}+W_{B} \Phi_{B}\right\}+\Xi\left\{\Phi_{0}-\Phi_{A}-\Phi_{B}\right\}
\end{aligned}
$$

with respect to its arguments $W_{A}, W_{B}, \Phi_{A}, \Phi_{B}, \Xi . \mathcal{Q}_{A}$ denotes the single chain partition function:

$\mathcal{Q}_{A}\left[W_{A}\right]=\frac{1}{V_{0}} \int \mathcal{D}_{1}[\mathbf{r}] \mathcal{P}_{1}[\mathbf{r}] e^{-\int_{0}^{1} \mathrm{~d} \tau W_{A}(r(\tau))}$

and similarly for $\mathcal{Q}_{B} ; \mathcal{Q}=\exp \left(\Delta \mu / 2 k_{B} T\right) \mathcal{Q}_{A}+\exp \left(-\Delta \mu / 2 k_{B} T\right) \mathcal{Q}_{B}$.

The values of $W_{A}, W_{B}, \Phi_{A}, \Phi_{B}, \Xi$ which extremize the free energy functional are denoted by lower-case letters and satisfy the self-consistent set of equations

$$
w_{A}(\mathbf{r})=\chi N \phi_{B}(\mathbf{r})-H(\mathbf{r}) N+\xi(\mathbf{r}) \quad \text { and } \quad \phi_{A}(\mathbf{r})=-\frac{V}{\mathcal{Q}} \frac{\mathcal{D} \mathcal{Q}_{A}}{\mathcal{D} w_{A}(\mathbf{r})}
$$

$\Phi_{0}(\mathbf{r})=\phi_{A}(\mathbf{r})+\phi_{B}(\mathbf{r})$, and similar expressions for $w_{B}$ and $\phi_{B}$. Substituting the extremal values of the densities and fields into the free energy functional (4) we calculate the free energy $G$ of the different phases. At coexistence the two phases have equal semigrandcanonical potential.

To calculate the monomer density we employ the end segment distribution which satisfies a diffusion equation. We expand the spatial dependence of the densities and fields in a set of orthonormal functions.[16,17] This procedure results in a set of non-linear equations which are solved by a Newton-Raphson-like method. We use up to 80 basis functions and achieve a relative accuracy $10^{-4}$ in the free energy.

The phase diagram in a thin film $\left(\Delta_{0}=0.9 R_{e}\right)$ with antisymmetric surface fields of various strengths is presented in Fig.1. For weak surface fields we find a second-order interface localization/delocalization transition.[13] 
Close to the critical point of the bulk enrichment layers form gradually at each surface and stabilize an interface in the center of the film. The films remains laterally homogeneous in this "soft-mode" phase. Only below the second order transition the interface is localized at one wall and $A$-rich and $B$-rich domains coexist laterally. Upon increasing the monomer-wall interaction strength the transition temperature shifts to lower values.
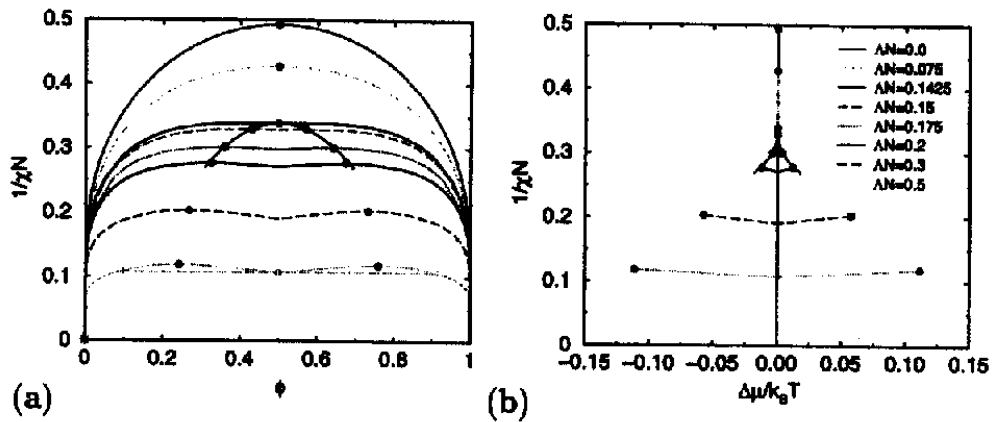

Figure 1. (a) Phase diagrams in a film with antisymmetric surface fields. The values of the surface fields $\Lambda N$ are indicated in the key. For $\Lambda N \leq 0.1425$ we find a single critical point, while we find two critical points for larger surface fields. (a) displays the phase diagram in the temperature-composition plane, while (b) presents the coexistence curves $\Delta \mu_{\text {coex }}(\chi N)$. (For $\Lambda N=0.15$ the two critical points are indistinguishable on the scale of panel (b)). From [13].

At large monomer-wall interaction the wetting transition is of first order and we observe also a first order interface localization/delocalization transition.[12,13] The prewetting coexistence slightly above the first order wetting transition temperature gives rise to two miscibility gaps in a film. At each wall a thin and a thick enrichment layer of the preferred component coexist. These coexistence regions terminate in two critical points which are the analogs of the prewetting critical points at each wall. When we decrease the temperature the miscibility gaps open and form a triple point at which an $A$-rich phase, a phase where the interface is delocalized in the middle of the film, and a $B$-rich phase coexist. Below the triple temperature an $A$-rich and a $B$-rich phase coexist.

The two types of phase diagrams are separated by a tricritical interface localization/delocalization transition. For our model we find a tricritical transition at $\Lambda N=0.1425$ and $\Delta_{0}=0.9 R_{e}$. When we increase the film width the tricritical strength of the surface interactions decreases. At tricriticality, there is only a single critical point, but the binodals are characterized by an exponent $\beta=1 / 4$ in mean field approximation.

Fig.1b presents the coexistence curves as a function of temperature and exchange potential. For a second order or a tricritical transition phase coexistence occurs at $\Delta \mu=0$ due to the symmetry of the system with respect to exchanging $A \rightleftharpoons B$. For a first order transition the coexistence curve lays on the symmetry axis $\Delta \mu=0$ for low temperatures. 
When the temperature rises, it bifurcates at the triple point; each continuation is the thin film analog of the prewetting line at the corresponding surface. Since the coexisting phases do not possess the symmetry of the Hamiltonian, $\Delta \mu_{\text {coex }} \neq 0$. For short range monomerwall interactions the prewetting-like curves deviate linearly from the bulk coexistence value[18] and terminate in critical points.
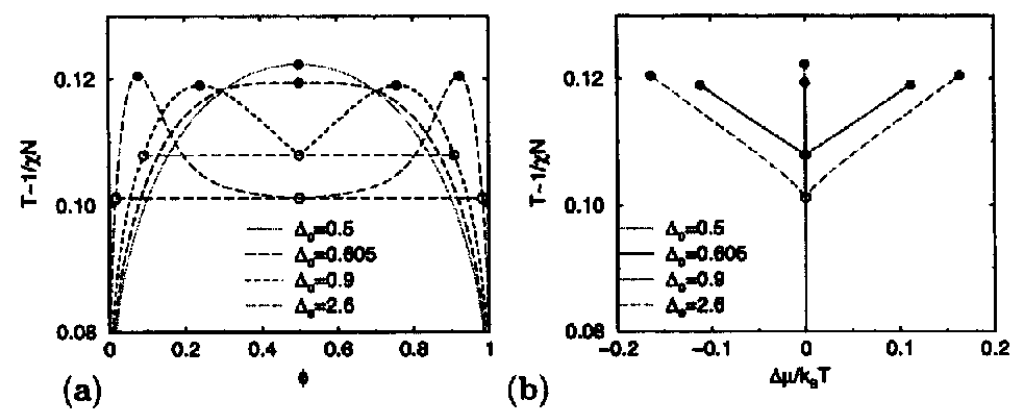

(b)

Figure 2. (a) Phase diagram for $\Lambda N=0.5$ and various film thicknesses $\Delta_{0}$. For $\Delta_{0}=$ $2.6 R_{e}$ and $0.9 R_{e}$ the interface localization-delocalization transition is first order, $\Delta_{0}=$ $0.605 R_{e}$ corresponds to a tricritical transition, while the transition is second order for $\Delta_{0}=0.5 R_{e}$. (b) Phase diagram as a function of temperature and chemical potential for the same parameters than in (a). From [12].

The interface localization/delocalization transition can also be changed from first to second order by decreasing the film thickness, $[8,12-14,19]$ because the effective interactions between the interface and each wall interfere. The three minimum structure of the effective interface potential close to the triple point changes to a single minimum as the film thickness becomes comparable to the range of the effective interaction between the interface and the wall. The film thickness dependence of the miscibility behavior is presented in Fig.2. For $\Delta_{0}<0.605 R_{e}$ the interface localization/delocalization transition is second order even though the wetting transition in the semi-infinite system is first order.

Of course, surface interactions in experimental realizations are never strictly antisymmetric or symmetric and it is important which degree of asymmetry is permissible without loosing the qualitative features of the limiting cases. The phase diagram for nonsymmetric boundary fields is discussed in Fig.3. The right walls attracts the $A$-component of the mixture and the surface fields lead to a first order wetting transition in the semiinfinite system. The monomer-wall interactions at the opposite wall are tuned from attracting $A$ (symmetric boundaries, capillary condensation) to attracting $B$ (antisymmetric boundaries, interface localization/delocalization).[11]

For symmetric boundaries the critical point is shifted to lower temperatures and higher concentration of the $A$ species attracted by both surfaces. The coexistence value of the chemical potential $\Delta \mu_{\text {coex }}$ is shifted to values disfavoring the $A$ component and the shift 
is roughly proportional to the inverse film widths (Kelvin equation). Above the wetting transition temperature an almost pure $A$-rich phase coexist with a $B$-rich phase. In the latter phase, there are thick enrichment layers of $A$ at the walls and the $B$ component prevails at the center of the film. When the temperature is lowered towards the wetting transition temperature $T_{\text {wet }}$, the thickness of the enrichment layers rapidly decreases. This gives rise to a convex curvature of the $B$-rich binodal slightly above $T_{\text {wet }}$. Below $T_{\text {wet }}$ the coexisting phases are almost pure, i.e., the enrichment layers in the $B$-rich phase are negligible and $\Delta \mu_{\text {coex }}$ is independent of temperature.
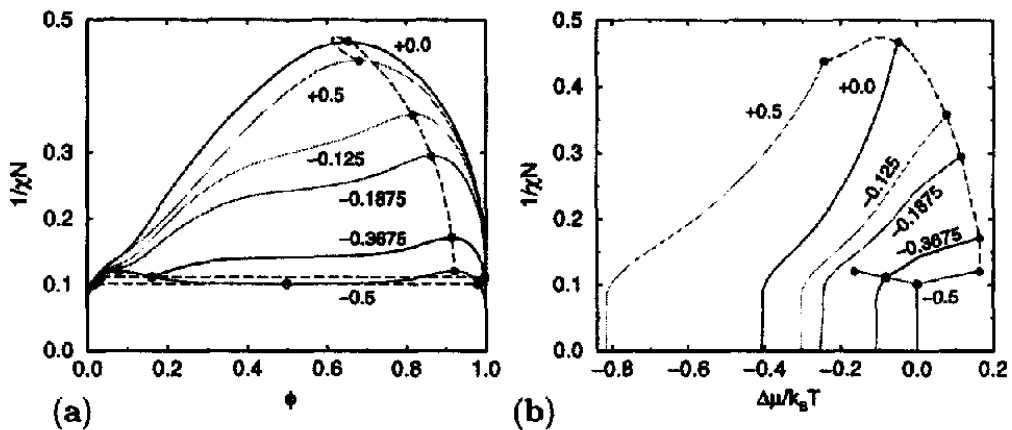

(b)

Figure 3. (a) Binodals for $\Delta_{0}=2.6 R_{e}$ and $\Lambda_{1} N=0.5 . \Lambda_{2} N$ varies as indicated in the key. The dashed curve shows the location of the critical points. Filled circles mark critical points, open circles/dashed horizontal lines denote three phase coexistence for $\Lambda_{2} N=-0.3675$ and -0.5 . The inset presents part of the phase boundary for antisymmetric boundaries. (b) Coexistence curves in the $\chi N-\Delta \mu$ plane. $\Lambda_{2} N$ varies according to the key. The "quasi-prewetting" lines for $\Delta \mu<0$ and $\Lambda_{2} N=-0.3675$ and -0.5 are indistinguishable, because they are associated with the prewetting behaviour of the surface with interaction $\Lambda_{1} N=+0.5$. From [11]

As we reduce the preference of the left wall for the $A$ component the system becomes more symmetric and the critical point shifts to more symmetrical composition and higher temperature (i.e., closer to the bulk critical point at $\chi N=2$ and $\phi=1 / 2$ ). If we make the left wall repulsive for the $A$ component (attractive for the $B$-component) the character of the transition gradually changes from a bulk-like unmixing transition, where the composition of the two phases varies little spatially across the film but differs between the two phases, to a prewetting-like transition, where an interface runs parallel to the walls and the distance between the wall and the interface is the order parameter. As this change occurs the critical temperature (composition) passes through a maximum (minimum). Upon approaching the strictly antisymmetric limit the coexistence curve (at low temperatures) approaches the bulk value. When it intersects with the prewetting line of the left wall, which attracts the $B$-species, a second two phase region opens between a thin and a thick enrichment layer at the left wall. 


\section{MONTE CARLO SIMULATIONS}

The self-consistent field calculations presented in the previous section neglect fluctuations. In a cylindrical pore, fluctuations destroy a true phase transition. In a thin film they change the universality class of the critical points from 3D Ising-like in the bulk to $2 \mathrm{D}$ Ising-like in a film. Moreover, there is an strong interplay between the wetting behavior of the semi-infinite system and the phase diagram of a film with antisymmetric boundaries and interface fluctuations (i.e., capillary waves) might modify the predictions of the mean field theory.

Being interested in the universal features of the phase diagram we have investigated a coarse grained lattice model of a binary polymer blend[20] via Monte Carlo simulations. In the framework of the bond fluctuation model[21] each monomer occupies the corner of a unit cell of a simple cubic lattice from further occupation. Monomers along the polymer are connected via bonding vectors of length $2, \sqrt{5}, \sqrt{6}, 3$ or 10 in units of the lattice spacing. Monomers interact through a square well potential which comprises the 54 nearest sites on the lattice. A contact between like monomers lowers the energy of the system by an amount $\epsilon$ (measured in units of $k_{B} T$ ), while a contact between different species increases the energy by the same amount. These interactions set a temperature scale and lead to a liquid--liquid phase separation at $T=1 / \epsilon_{c}=69.3(3)$ in the bulk.[22] The parameter $\epsilon$ is related to the Flory-Huggins parameter via $\chi=2 z_{c} \epsilon$, where $z_{c}=$ 2.65 denotes the number of monomers of other chains in the range of the square well potential.[20]

We work at a chain length $N=32$ and monomer number density $\rho=1 / 16$. The molecules end-to-end distance is $R_{e} \approx 17$ in units of the lattice spacing. We study thin film of geometry $L \times L \times \Delta$. Periodic boundary conditions are applied in the two lateral directions, while there are hard impenetrable walls a distance $\Delta$ apart. Monomers in the two layers nearest to the walls interact with the boundaries. An $A$-monomer close to the right wall decreases the energy by $\epsilon_{w}=0.16$, while a $B$-monomer increases the energy by the same amount. For symmetrical walls the interactions at the left wall are identical to the right wall; for antisymmetrical walls $B$-monomers are attracted by the left wall and $A$-monomers repelled. Using the Young equation we have determined the wetting transition temperature to $T_{\text {wet }}=14.1(7)$.[23] The wetting transition occurs well inside the strong segregation limit and is of first order.

We use the semi-grandcanonical ensemble, i.e., we fix the temperature and the exchange potential $\Delta \mu$ and monitor the composition $\phi$ and its fluctuations. In addition to Monte Carlo moves which update the conformations of the polymers on the lattice (local hopping attempts and slithering-snake-like motions) we try to change the chain species $A \rightleftharpoons B$ at fixed conformation. This semi-grandcanonical simulation scheme is employed in junction with reweighting methods as to encourage the system to sample all compositions with roughly equal probability.

In the simulations we monitor the joint distribution of the composition, energy and monomer-wall interaction; and we use finite size scaling techniques and histogram reweighting methods to locate the critical point. As an example Fig.4, presents the probability distribution of the composition as a function of the film thickness in the vicinity of the tricritical point. The distribution shows a clear three peak structure. The tempera- 


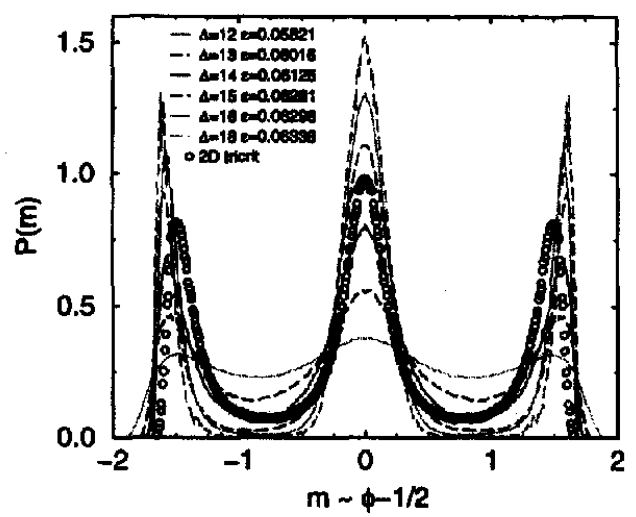

Figure 4. Probability distribution of the composition scaled to unit norm and variance for various film thickness $\Delta\left(R_{e}=17\right.$ in units of the lattice spacing) and temperatures as indicated in the key. The probability distribution of the $2 \mathrm{D}$ tricritical universality class is represented by circles. From [14]

ture is adjusted such that the ratio of the heights of the central and outer peaks equals 1.2. This value corresponds to the ratio of the distribution of the tricritical universality class. The latter quantity has been measured in simulations of the Blume-Capel model at its tricritical point.[24] To compare the distributions without adjustable parameter we scale them to unit norm and variance. For film thickness $\Delta=14 \approx 0.82 R_{e}$ the shape of the distribution matches closely the universal function and this holds also true for larger systems. For $\Delta<0.82 R_{e}$ we find a second order interface localization/delocalization transition, while there is a first order transition for $\Delta>0.82 R_{e}$.

The probability distribution of the composition yields information about the interaction $g(l)$ between the walls and the interface. Since the wetting transition occurs in the strong segregation limit, the coexisting phases in the bulk are almost pure $\phi_{\text {coex }}^{\text {bulk }} \approx 0$ or 1 and "bulk"-like composition fluctuations can be neglected. Hence, the distance $l$ between the wall and the interface is given by $l=\Delta \phi$ and the effective interface potential $g(l)$ can be measured in the Monte Carlo simulations according to $g(l)=-k_{B} T / L^{2} \ln P(\phi)$, where $P(\phi)$ denotes the probability distribution of the composition.

The result for the effective interface potential $g(l)$ in the vicinity of the triple point is presented in Fig.5. The three minima correspond to the $A$-rich phase, the phase with the delocalized interface, and the $B$-rich phase. Unlike the situation at the tricritical point the position of the minima does not depend on the lateral system size. However, the effective interface potential does depend on $L$; the minima broaden upon increasing the lateral system size $L$ and the free energy of the delocalized state decreases with respect to the localized ones. The dependence of $g(l)$ on the lateral system size gives rather direct evidence for a renormalization of the effective interface potential by interface fluctua- 


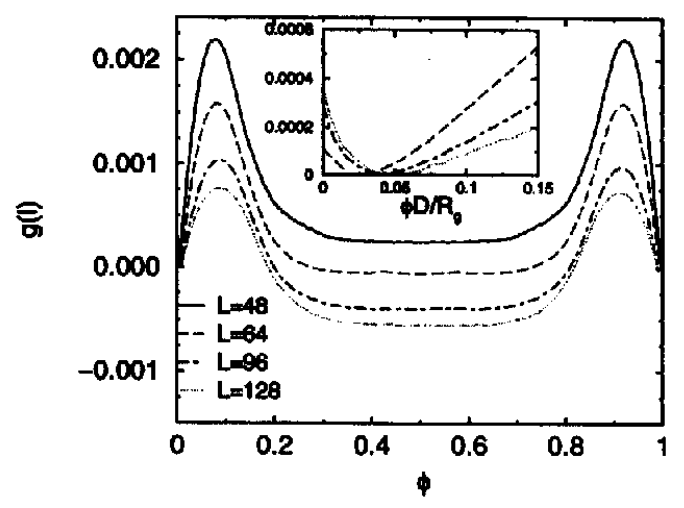

Figure 5. Dependence of the effective interface potential $g(l) / k_{B} T$ on the lateral film extension $L$ for $\Delta=48 \approx 2.8 R_{e}$ and $\epsilon=0.069$. The inset presents an enlarged view on the minimum of the localized state. From [14]

tions $[25,26]$ in the framework of a microscopic model. The interface in the simulations is not ideally flat, but there are long wavelength fluctuations of the local interface position (i.e., capillary waves). Since the interface is not unconstraint, the interface potential imparts a parallel correlation length onto the capillary waves. $\xi_{\|} \sim \sqrt{\mathrm{d} g^{2} / \mathrm{d}^{2} \phi}$. This parallel correlation length is larger in the delocalized state than in the localized one. For the parameters of the simulation the lateral system size and the parallel correlation lengths are of the same order of magnitude. For lateral distances smaller than $\xi_{\|}$the local position fluctuates like a free interface, for lateral distance that exceed $\xi_{\|}$interface fluctuations are strongly suppressed.[27] In the simulations the lateral system size $L$ also cuts off interface fluctuations when $L<\xi_{\|}$. [28] Interface fluctuations reduce the free energy of the system and the minima corresponding to the delocalized state benefits more from an increase of the lateral system size. This rationalized qualitatively the effect observed in Fig.5 and quantitative description shall be presented elsewhere.[14] The effect is important for accurately locating the triple temperature.

The phase diagrams for $\Delta \approx 2.8 R_{e}$, as revealed by Monte Carlo simulations in Fig.6, confirm the qualitative predictions of the self-consistent field calculations. For symmetric boundary conditions there is a small shift of the critical point to lower temperatures and higher concentration of the $A$ species preferred by the walls. The "bulge" of the $A$-poor binodal is a consequence of the vicinity of the prewetting critical point.[23] If the film thickness were larger the coexistence value $\Delta \mu_{\text {coex }}$ would be smaller (Kelvin equation) and the coexistence curve would intersect the prewetting line. In this case there would be a two phase coexistence region also in the case of symmetric boundaries. $[23,29]$ In the antisymmetric case the phase diagram comprises two critical points - the analogs of the prewetting critical points at each surface. The concomitant miscibility gaps joint to form a triple point ultimately above the temperature of the first order wetting transition 

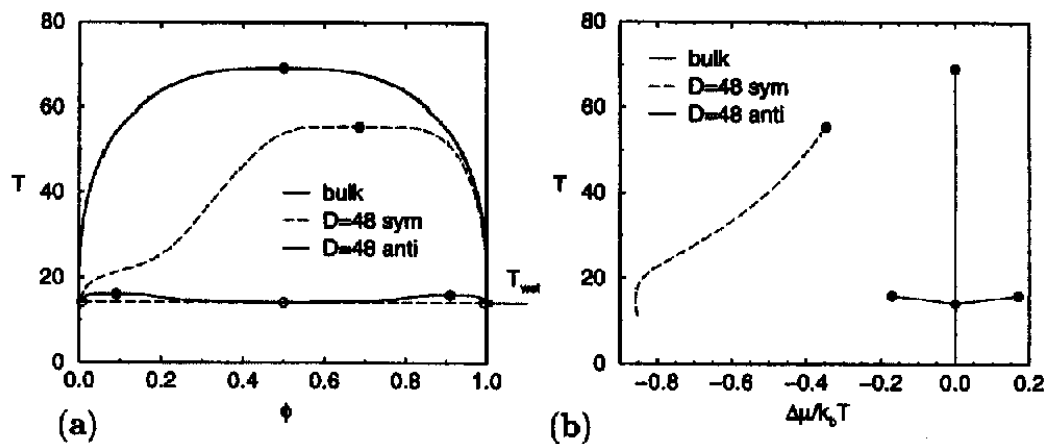

(b)

Figure 6. Phase diagram in terms of composition and temperature (a) and exchange potential and temperature (b) for film thickness $\Delta 48 \approx 2.8 R_{e}$. From [14]

temperature of the semi-infinite system. Unlike the mean field predictions, however, the shape of the binodals is much flatter reflecting the 2D Ising behavior close to the critical points.

\section{DISCUSSION}

We have calculated the phase diagram of a symmetric polymer mixture confined into a thin film in mean field approximation and by Monte Carlo simulations. The mean field calculations reveal a rich interplay between the phase behavior in confined geometry and the wetting behavior of the semi-infinite system and the general features of the phase behavior are confirmed by our Monte Carlo results. However, fluctuations result in two modifications of the mean field results: (i) In the vicinity of the critical point we observe 2D Ising critical behavior with much flatter binodals than the parabolic binodals of the mean field universality class. (ii) The effective interface potential is renormalized by capillary waves. This leads, e.g., to a systematic overestimation of the triple temperature by the mean field treatment.

Qualitatively the interplay between the prewetting behavior and the phase diagram in a film with antisymmetric boundaries is not specific to polymer blends but is rather characteristic of all binary mixtures. We expect, however, polymer mixtures, be particularly suitable model systems for exploring these effects experimentally.

If we measure the incompatibility of the species/temperature by $\chi N$ and the length scale in units of the end-to-end distance $R_{e}=b \sqrt{N}$, the typical scale of the free energy in a volume $R_{e}^{3}$ is given by $\sqrt{N}$, where where the reduced chain length $\bar{N}=\left(\rho R_{e}^{3} / N\right)^{2}$ measures the degree of interdigitation.

The importance of fluctuations close to the critical point can be gauged by the Ginzburg criterium. For binary polymer blends one finds that fluctuations are important in the range $\left|1-\chi_{c} N / \chi N\right| \ll G i$ with Ginzburg number $G i \sim 1 / \bar{N}$. The importance of interface 
fluctuations can be described by the capillary parameter[30,31]

$\omega=\frac{k_{B} T \lambda^{2}}{4 \pi \sigma_{A B}}=\frac{1}{4 \pi \sqrt{\bar{N}}}\left(\lambda R_{e}\right)^{2} \frac{k_{B} T \sqrt{\bar{N}}}{\sigma R_{e}^{2}}$

where $1 / \lambda$ denotes the characteristic length of the interface potential and $\sigma$ is the interface tension. $\lambda R_{e}$ and $\sigma R_{e}^{2} / \sqrt{N} k_{B} T$ are functions of $\chi N$ only. Upon increasing the degree of interdigitation $\bar{N}$, the capillary parameter $\omega \sim 1 / \sqrt{\bar{N}}$ decreases.

In the limit of long chains (or strong interdigitation) these fluctuation effects are suppressed and the self-consistent field theory is believed to describe the confined blend quantitatively appropriately. For the bond fluctuation model $N=32$ corresponds to $\bar{N} \approx 91$ and this chain length of our coarse grained model corresponds roughly to $100-150$ repeat units of a real polymer. Since fluctuations do not alter the qualitative mean field scenario for the rather short chains investigated by Monte Carlo simulations, we expect this a fortiori for experimental systems. Furthermore the wetting transition in binary polymer mixtures occurs far below the critical point. Bulk-like composition fluctuations are only of minor importance; the systems are well describable via effective interface Hamiltonians and the effect of interface fluctuations is clearly observable. Additionally, the size of the enrichment layers is set by $R_{e}$ and, hence, is much larger than in atomic liquids. Sophisticated profiling techniques (e.g., nuclear reaction analysis, neutron reflectometry or secondary ion mass spectrometry) are available to accurately determine the thickness of wetting layers in experiments.

Indeed, experiments on polymer mixtures were the first to investigate fluctuation effects in the delocalized state[32,33] and the wetting transition in polymer blends has been observed experimentally. [34,35] Our results imply, for instance, that ultrathin enrichment layers with a thickness $l \approx R_{e} / 5$ be unstable in the temperature range $T_{\text {wet }}<T<T_{\text {prewet }}$. Such instabilities slightly above $T_{\text {wet }}$ have been observed for compressible one-component polymer films.[36]

Of course, both the self-consistent field calculations and the Monte Carlo simulations deal with highly idealized models. The effect of architectural asymmetry between the constituents of the mixture or the role of long-range van der Waals interactions between the monomers and the surfaces has not be considered.

\section{ACKNOWLEDGMENT}

It is a great pleasure to thank N.B. Wilding for providing the scaling function of the 2D tricritical universality class (Fig.4). Financial support was provided by the DFG under grant Bi314/17 in the priority program "wetting and structure formation at interfaces" and by the DAAD/PROALAR2000.

\section{REFERENCES}

1. R. Evans, J.Phys.Cond.Matter 2, 8989 (1990).

2. A.O. Parry, J.Phys.Condens.Matter 8, 10761 (1996).

3. S. Dietrich, Phase Transitions and Critical Phenomena, Vol 12, C. Domb and J. Lebowitz (eds) Academic Press, London (1988). 
4. M.E. Fisher and H. Nakanishi, J.Chem.Phys. 75, 5857 (1981). H. Nakanishi and M.E. Fisher, J.Chem.Phys. 78, 3279 (1983).

5. L.D. Gelb et al., Rep.Prog.Phys. 62, 1573 (1999).

6. F. Brochard-Wyart and P.-G. de Gennes, Acad.Sci. Paris 297, 223 (1983).

7. A.O. Parry and R. Evans, Phys.Rev.Lett. 64, 439 (1990), Physica A 181, 250 (1992).

8. M.R. Swift at al., Europhys.Lett. 14, 475 (1991).

9. K. Binder et al., Phys.Rev.Lett. 74, 298 (1995); Phys.Rev. E 51, 2823 (1995). E.V. Albano et al., Surf.Sci. 233, 151 (1989).

10. K. Binder et al., Phys.Rev. E 53, 5023 (1996).

11. M. Müller et al., Europhys.Lett. 49, 724 (2000).

12. M. Müller et al., Physica A 279, 188 (2000).

13. M. Müller et al., Phys.Rev. E (in press).

14. M. Müller and K. Binder, (to be submitted).

15. J. Noolandi, K.M. Hong, Macromolecules 14, 727 (1981).

16. M.W. Matsen, Phys.Rev.Lett. 74, 4225 (1995); M.W. Matsen and M. Schick, Phys.Rev.Lett. 72, 2660 (1994).

17. M.W. Matsen, J.Chem.Phys. 106, 7781 (1997); T. Geisinger et al., J.Chem.Phys. 111, 5241 (1999).

18. E.H. Hauge and M. Schick, Phys.Rev. B 27, 4788 (1983).

19. A.M. Ferrenberg at al., Phys.Rev. E 58, 3353 (1998).

20. M. Müller, Macromol. Theory Simul. (Feature Article) 8, 343 (1999).

21. I. Carmesin and K. Kremer, Macromolecules 21, 2819 (1988).

22. H.-P. Deutsch and K. Binder, Macromolecules 25, 6214 (1992).

23. M. Müller and K. Binder, Macromolecules 31, 8323 (1998).

24. N.B. Wilding and P. Nielaba, Phys. Rev. E 53, 926 (1996).

25. R. Lipowski et al., Phys.Rev. B 27, 499 (1983).

26. E. Brezin et al., Phys.Rev.Lett. 50, 1387 (1983).

27. M. Schick, Liquids at Interfaces, Les Houches, Session XLVIII, J. Charvolin, J.F. Joanny, and J. Zinn-Justin (eds), Elsevier, Amsterdam (1990).

28. A. Werner et al., J.Chem.Phys. 107, 8175 (1997).

29. D. Nicolaides and R. Evans, Phys.Rev. B 39, 9336 (1989). R. Evans and U. Marini Bettolo Marconi, Phys. Rev. A 32, 3817 (1985).

30. D.S. Fisher and D.A. Huse, Phys.Rev. B 32, 247 (1985).

31. M.E. Fisher and H. Wen, Phys.Rev.Lett. 68, 3645 (1992).

32. T. Kerle et al, Phys.Rev.Lett. 77, 1318 (1996), Europ. Phys. J. B7, 401 (1999).

33. M. Sferrazza at al., Phys.Rev.Lett. 81, 5173 (1998);ibid 78, 3693 (1997).

34. J. Rysz et al., Europhys.Lett. 50, 35 (2000).

35. M. Geoghegan et al., Phys.Rev. E 62, 940 (2000).

36. W. Zhao et al., Phys.Rev.Lett. 70, 1453 (1993). 